\title{
Probing the mechanism of high capacitance in two-dimensional titanium carbide using in-situ X-Ray absorption spectroscopy
}

Maria R. Lukatskaya, ${ }^{\dagger}$ Seong-Min Bak, ${ }^{\dagger}$ Xiqian Yu, Xiao-Qing Yang, * Michel W. Barsoum, and Yury Gogotsi*

M. R. Lukatskaya, Prof. M.W. Barsoum, Prof. Y. Gogotsi

Department of Materials Science and Engineering and A.J. Drexel Nanomaterials Institute, Drexel University, Philadelphia, PA 19104, USA

E-mail: gogotsi@drexel.edu

Dr. S.-M. Bak, Dr. X. Yu, Dr. X.-Q. Yang

Chemistry Department, Brookhaven National Laboratory, Upton, New York 11973, USA

E-mail: xyang@bnl.gov

Keywords: capacitance, two-dimensional, carbide, MXene, XAS, pseudocapacitance

The field of supercapacitors (electrochemical capacitors) is constantly evolving. The global motivation is to create devices that possess a significant energy density without compromising the power density ${ }^{[1]}$. To achieve this goal, new materials must be discovered and complex electrode architectures developed ${ }^{[2]}$.

Several decades ago, impressive electrochemical properties of the hydrated ruthenium oxide were discovered. ${ }^{[3]}$ Important features of $\mathrm{RuO}_{2}$ are: i) good conductivity, which is not typical of transition metal oxides, and, ii) surfaces that participate in reversible redox reactions during charge/discharge, enabling capacitance of 1000 to $1800 \mathrm{~F} / \mathrm{cm}^{3}$ for thick ( $>20$ $\mu \mathrm{m})$ electrodes ${ }^{[4]}$ and up to $2800 \mathrm{~F} / \mathrm{cm}^{3}$ for thin films $(50-100 \mathrm{~nm}) \cdot{ }^{[5]}$ However, the high cost of this material, along with a significant decline in charge storage capacitances with increased electrode thicknesses outweighs its outstanding volumetric capacitance, rendering this material unsuitable for applications beyond microscale energy storage. ${ }^{[4 a]}$

In search for alternatives, MXenes - a new but quickly expanding family of twodimensional transition metal carbides $\left(\mathrm{Nb}_{2} \mathrm{C}, \mathrm{Ti}_{3} \mathrm{C}_{2}, \mathrm{Ta}_{4} \mathrm{C}_{3}\right.$, etc.), captured the attention of researchers recently. ${ }^{[6],[7]}$ MXenes possess excellent conductivity, and offer a broad variety of chemistries. $^{[7 \mathrm{a}]}$ MXenes are produced by the selective removal of the atomically thin aluminum layers from the MAX phase ${ }^{[8]}$ structure, e.g., $\mathrm{Ti}_{3} \mathrm{AlC}_{2}$, via wet chemistry routes, 
such as etching in $\mathrm{HF},{ }^{[9]} \mathrm{NH}_{4} \mathrm{HF}_{2}{ }^{[10]}$ or acidic solutions of fluoride salts, i.e. $\mathrm{LiF}$ in $\mathrm{HCl}{ }^{[11]}$ The described treatments result in mixed fluorine- and oxygen-containing terminations of transition metal atoms on the MXene surfaces. Thus, the general formula describing MXene composition is $M_{n+1} X_{n} T_{x}$, where $M$ is an early transition metal, $X$ is carbon and/or nitrogen, $n$ $=1,2$ or $3, \mathrm{~T}$ is surface termination $(-\mathrm{F},-\mathrm{OH},=\mathrm{O})$ and $\mathrm{x}$ is number of surface groups per formula unit ${ }^{[7 a]}$.

This combination of terminated transition metal on the surface with a metallically conductive carbide core is believed to be key to the MXenes' promising performance as electrode materials for energy storage applications, such as Li-ion batteries, ${ }^{[12]} \mathrm{Li}-\mathrm{S}$ batteries $^{[13]}$ and supercapacitors. ${ }^{[6]}$ In particular, impressively high volumetric capacitance reaching $900 \mathrm{~F} / \mathrm{cm}^{3}$ (comparable only with hydrated $\mathrm{RuO}_{2}$ ) was measured for $\mathrm{Ti}_{3} \mathrm{C}_{2} \mathrm{~T}_{\mathrm{x}}$ in aqueous electrolytes. ${ }^{[11,14]}$ Our previous studies revealed that spontaneous intercalation of some cations takes place when MXene samples are exposed to aqueous electrolytes. ${ }^{[6]}$ In-situ XRD showed that variations of the distance between the $\mathrm{Ti}_{3} \mathrm{C}_{2} \mathrm{~T}_{\mathrm{x}}$ layers (which is proportional to the $c$ lattice parameter, $c$-LP) during cycling is small $(<5 \%)$ and no phase changes were detected. ${ }^{[6]}$ Electrochemical quartz crystal admittance (EQCA) studies of $\mathrm{Ti}_{3} \mathrm{C}_{2} \mathrm{~T}_{\mathrm{x}}$ multilayer particles in several aqueous electrolytes with different cations revealed that cation insertion is accompanied by electrode deformations (expansion/contraction), facilitated by the presence of water molecules. These deformations occur so quickly, however, that they closely resemble 2D ion adsorption at solid-liquid interfaces. ${ }^{[15]}$ Moreover, it was found that surface chemistry has a significant effect on the capacitive response in acids: decreased amount of Fterminations, relative to those of $\mathrm{O}$ and $\mathrm{OH}$, resulted in a significant increase in specific capacitance, i.e. almost doubling it. ${ }^{[14]}$

These results notwithstanding, the understanding of what fundamental processes are responsible for the MXenes promising performance has been lacking. While double layer ion adsorption can be ruled out as the main mechanism, due to the relatively low specific surface 
area of multi-stacked MXenes, ${ }^{[6]}$ possible pseudocapacitive storage mechanisms can be hydrogen storage or redox coupled capacitance of MXene (Figure 1a). In this communication, we report on a set of electrochemical experiments coupled with in-situ X-ray absorption spectroscopy (XAS) that shed light on the mechanism of capacitance in $\mathrm{Ti}_{3} \mathrm{C}_{2} \mathrm{~T}_{\mathrm{x}}$ MXene.

Typical electrochemical performance of the MXenes can be characterized by the nearly rectangular cyclic voltammetry loops as shown in Figure $\mathbf{1 b}$ for $\mathrm{Ti}_{3} \mathrm{C}_{2} \mathrm{~T}_{\mathrm{x}}$ MXene (produced by etching of $\mathrm{Ti}_{3} \mathrm{AlC}_{2} \mathrm{MAX}$ phase in the $\mathrm{LiF}-\mathrm{HCl}$ solution) ${ }^{[11]}$ in $1 \mathrm{M} \mathrm{H}_{2} \mathrm{SO}_{4}$. To ensure that the observed electrochemical behavior is not due to parasitic reactions and also to evaluate possible contributions from hydrogen evolution, a series of constant potential experiments during which the current change with time was monitored (Figure 1c) - were performed. It is well established that di-hydrogen $\left(\mathrm{H}_{2}\right)$ evolution is a faradic process and its intensity (i.e., current) is dependent on the over-potential value. By collecting chronoamperometry (CA) data, at various fixed potentials, a better understanding of the underlying processes can be obtained - as compared to the cyclic voltammetry (CV), which is unlike CA is dynamic: the sample is subjected to a certain potential for no more than several seconds per cycle.

Based on the observed current density values (Figure 1c), we categorized the potentials at which the CA was performed into three regimes: i) a "safe" region where the current density does not exceed $\sim 0.1 \mathrm{~A} / \mathrm{g}$ (marked in green in Figure 1c), and no $\mathrm{H}_{2}$ evolution is observed, ii) a $\mathrm{H}_{2}$-“storage” region, where the current density is in the 0.1-0.3 A/g range, (marked in yellow in Figure 1c), and where no noticeable bubble formation is observed. In this region, the CA profiles are smooth and it can be used when the electrode is cycled at scan rates of $>\sim 20 \mathrm{mV} / \mathrm{s}$, and, iii) a $\mathrm{H}_{2}$-evolution region (marked pink in Figure 1c), where the current density $>0.3 \mathrm{~A} / \mathrm{g}$ and active $\mathrm{H}_{2}$ evolution is observed. The onset of hydrogen evolution (beginning of Regime 3) limits the cathodic potential for the use of MXene electrode. It is worth noting, that this approach of selecting a voltage window and the estimating contributions of various parasitic reactions is applicable to other supercapacitor 
materials/devices as well. In this case, it allowed us to evaluate the extent of $\mathrm{H}_{2}$ evolution by simply measuring the current transients, where $\mathrm{H}_{2}$ bubble formation is mirrored by an uneven current profile. It follows, that the lowest negative, and "safe", voltage limit for $\mathrm{Ti}_{3} \mathrm{C}_{2} \mathrm{~T}_{\mathrm{x}}$ in 1 $\mathrm{M} \mathrm{H}_{2} \mathrm{SO}_{4}$ is $-0.35 \mathrm{~V}$ vs. Ag/AgCl.

As noted above, one of the potential mechanisms for high capacitance is electrochemical hydrogen storage, as has been shown for some carbon materials. ${ }^{[16]}$ However, it is unlikely to have a significant contribution due to i) acidity of the electrolyte used: it was reported that hydrogen storage has significant contribution to energy storage only in basic electrolytes, and ii) low specific surface area of MXenes. ${ }^{[16]}$

We now turn our attention to intercalation pseudocapacitance, i.e. redox processes in the $\mathrm{Ti}_{3} \mathrm{C}_{2} \mathrm{~T}_{\mathrm{x}}$ during cycling. If that is the case, then $\mathrm{Ti}$ atoms would be changing their oxidation state during electrochemical cycling.

In order to investigate it, we performed electrochemical in-situ X-ray absorption near edge structure spectroscopy (XANES) for the Ti K-edge. It is well known that XANES is a powerful technique to probe oxidation states of elements, it was successfully applied in the past to shed light on the storage mechanism of $\mathrm{MnO}_{2}{ }^{[17]}$ and $\mathrm{RuO}_{2}{ }^{[18]}$ by tracking changes in metal edge position during electrochemical cycling. Herein, we monitored the shifts in the Ti edge energy at different points of the charge and discharge. In situ Ti K-edge XAS spectra were collected - for a sequence of applied potentials - starting from 0.275 and ending at -0.35 $\mathrm{V}$, and then from -0.2 to $0.345 \mathrm{~V}$ on reverse scan, as shown on the CV curve in Figure 2a. The Ti K-edge XANES spectra presented in Figure $\mathbf{2 b}$ and $\mathbf{c}$ feature a relatively weak preedge peak 'A' at $4971 \mathrm{eV}$, and a strong main absorption peak 'B' at around $4985 \mathrm{eV}$. The weak pre-edge absorption involves the hybridization of the metal $3 d$ and carbon $2 p$ orbitals. ${ }^{[19]}$ That peak 'A' can be assigned to the transition of a 1s electron to the hybridized $\mathrm{t}_{2 \mathrm{~g}}(\mathrm{Ti} 3 d+\mathrm{C} 2 p)$ and $\mathrm{e}_{\mathrm{g}}(\mathrm{Ti} 3 d+\mathrm{C} 2 p)$ orbitals. The main absorption peak 'B', however, is due to the dipole allowed transition of 1 s electrons to unoccupied Ti $4 p$ states. 
Although the spectra did not show significant difference in the shape, the edge shifts are clearly recognized in both charge and discharge potential sweeps (insets in Figure $2 \mathrm{~b}$ and c). A plot of the Ti K-edge energies, at half height of normalized XANES spectra, versus applied potential (Figure 2d) clearly shows the changes at each potential. Sweeping from 0.275 to $0.35 \mathrm{~V}$, shifts the Ti edge to lower energies. Such a shift is consistent with a decrease in the average oxidation state of the $\mathrm{Ti}$ atoms. During the reverse scan to $0.345 \mathrm{~V}$, the XANES spectrum shifts back to the higher energy (and higher Ti oxidation state).

This moderate edge shift in the XANES energy - corresponding to a reduction/oxidation of the $\mathrm{Ti}$ - is consistent with our previous study of $\mathrm{Ti}_{3} \mathrm{C}_{2} \mathrm{~T}_{\mathrm{x}}$ in Li-ion batteries. ${ }^{[20]}$ In that case, an edge shift of $\approx 0.6 \mathrm{eV}$ was observed over a potential window of $\approx 3.0 \mathrm{~V}\left(\mathrm{vs} . \mathrm{Li}^{+} / \mathrm{Li}\right.$ ) during lithiation (i.e., reduction of Ti). ${ }^{[20]}$

There is clear change in Ti oxidation state during cycling. To quantify the change, we plotted the Ti K-edge XANES spectra of $\mathrm{Ti}_{3} \mathrm{C}_{2} \mathrm{~T}_{\mathrm{x}}$ at applied potentials (i.e., $-0.4 \mathrm{~V}$ and $0.1 \mathrm{~V}$ ) together with the reference Ti oxides (i.e., $\mathrm{TiO}$ and anatase $\mathrm{TiO}_{2}$ ) in Figure 3a. Based on the linear dependence between edge energy and $\mathrm{Ti}$ average oxidation state ${ }^{[21]}$, the latter can be estimated in the MXene sample during cycling (Figure 3b). From the inset shown in Figure 3b, we see that the average oxidation state of Ti changes from 2.33 to 2.43 (i.e. by $\approx 0.1 \bar{e}$ per $\mathrm{Ti}$ atom) over a $0.7 \mathrm{~V}$ window.

To explore the viability of this value we estimated capacitance using the following formula: $C_{g}=F \cdot \delta /(M W \cdot V)$, where $C_{g}[\mathrm{~F} / \mathrm{g}]$ stands for gravimetric capacitance, $F$ [96485 $\mathrm{C} / \mathrm{mol}$ ] is a Faraday constant, $\delta$ is number of electrons participating in electrochemical reaction (equal to $0.1 \cdot 3=0.3$ in our case since there are $3 \mathrm{Ti}$ atoms in $\mathrm{Ti}_{3} \mathrm{C}_{2} \mathrm{~T}_{\mathrm{x}}$ ), $M W[\mathrm{~g} / \mathrm{mol}]$ is molar weight, $V[\mathrm{~V}]$ is voltage window ( $0.7 \mathrm{~V}$ in this case) and assumed electrochemical reaction is:

$$
\mathrm{Ti}_{3} \mathrm{C}_{2} \mathrm{O}_{\mathrm{x}}(\mathrm{OH})_{\mathrm{y}} \mathrm{F}_{2-\mathrm{x}-\mathrm{y}}+\delta \bar{e}+\delta \mathrm{H}^{+} \rightarrow \mathrm{Ti}_{3} \mathrm{C}_{2} \mathrm{O}_{\mathrm{x}-\delta}(\mathrm{OH})_{\mathrm{y}+\delta} \mathrm{F}_{2-\mathrm{x}-\mathrm{y}}
$$


Since the molar weights for O (16), OH (17) and F (19) are close, the overall formula weight can be assumed to be $202 \mathrm{~g} / \mathrm{mol}$. Thus we get specific capacitance values of $205 \mathrm{~F} / \mathrm{g}$. These values are in a good agreement with the experimental specific capacitances measured herein for $\mathrm{Ti}_{3} \mathrm{C}_{2} \mathrm{~T}_{\mathrm{x}}$, which are $230 \mathrm{~F} / \mathrm{g}$ (when measured in Swagelok cell). Please note that in in situ XAS cell we measured specific capacitance of $~ 150$ F/g (for in situ experiments, a hole was drilled in the center of the cell and it was impossible to create a good contact between the electrode and the current collector).

In conclusion, we performed a thorough analysis of the electrochemical behavior of the $\mathrm{Ti}_{3} \mathrm{C}_{2} \mathrm{~T}_{\mathrm{x}}$ and analyzed factors contributing to MXene' capacitance. We demonstrated a procedure for the safe voltage window selection, which can be applied to other electrochemical systems and that provides an important insight into electrolyte decomposition processes. Electrochemical in situ XAS measurements detected changes in Ti oxidation state during cycling. Even though this method is semi-empirical and provides rough estimation, the estimated value is comparable with the value that we back-calculated from the sample's gravimetric capacitance assuming redox-only capacitance. Therefore, we can conclude that electrochemical behavior of the $\mathrm{Ti}_{3} \mathrm{C}_{2} \mathrm{~T}_{\mathrm{x}}$ MXene in sulfuric acid is predominantly pseudocapacitive. This makes $\mathrm{Ti}_{3} \mathrm{C}_{2} \mathrm{~T}_{\mathrm{x}}$ MXene one of very few examples of materials that exhibit "true" pseudocapacitive behavior: it presents a continuous change in titanium oxidation state during charge/discharge, producing rectangular-shaped CVs. This behavior can be attributed to $2 \mathrm{D}$ nature of $\mathrm{Ti}_{3} \mathrm{C}_{2} \mathrm{~T}_{\mathrm{x}}$ MXene: spontaneous ion intercalation naturally provides access to electrochemically active transition metal oxide surfaces, while the conductive carbide layer ensures rapid charge transfer. Those properties are not available in titanium oxide or cubic TiC. The results obtained herein are important for another reason: namely, they indicate, as a first approximation, that the average Ti oxidation state in $\mathrm{Ti}_{3} \mathrm{C}_{2} \mathrm{~T}_{\mathrm{x}}$ is much closer to +2 than +4 .

\section{Experimental Section}


Electrochemical measurements: Preliminary electrochemical measurements were performed in a 3-electrode Swagelok cell, with $\mathrm{Ag} / \mathrm{AgCl}$ as reference electrode, $6 \mathrm{mg} \mathrm{Ti}_{3} \mathrm{C}_{2} \mathrm{~T}_{\mathrm{x}}$ electrode (containing 5 wt. \% of PTFE binder and 5 wt. \% of carbon black) as working electrode and 33 mg AC (containing 5 wt. \% of PTFE binder) as counter electrode. During all measurements, the overall cell potential was monitored in order to controllably translate into the 2-electrode cell geometry used in the in situ XAS measurements.

In-situ X-ray Absorption Spectroscopy (XAS): In-situ Ti K-edge XAS measurement were performed at beamline X18A at the National Synchrotron Light Source (NSLS) of the Brookhaven National Laboratory (BNL) using a Si(111) double-crystal monochromator, detuned to $40 \%$ of its original maximum intensity to eliminate high-order harmonics.

The spectroscopic data was collected in fluorescence mode with a passivated implanted planar silicon (PIPS) detector in grazing incident geometry. The PIPS detector was placed normal to the incident beam and at a $45^{\circ}$ angle to the sample. The Ti K-edge energy, $4966 \mathrm{eV}$, was calibrated using the first inflection point of the edge region of a metallic Ti foil recorded before and after measurement.

For the in-situ XAS measurements, during electrochemical testing, we used a twoelectrode 2032 coin-type cells, with a hole (diameter: $3 \mathrm{~mm}$ ) drilled on the working electrode side. The MXene served as the working electrode, and the activated carbon as the counter electrode. A glass fiber separator (Whatman) was soaked in a $1 \mathrm{M} \mathrm{H}_{2} \mathrm{SO}_{4}$ aqueous electrolyte. The hole was sealed with a Mylar film.

Detailed information on sample and electrode preparation, electrochemical measurements can be found in Supporting Information file.

\section{Supporting Information}

Supporting Information is available from the Wiley Online Library or from the author.

\section{Acknowledgements}

${ }^{\dagger}$ M. R. Lukatskaya and S.-M. Bak contributed equally to this work. 
We thank Michael Ghidiu for help with material synthesis and Sankalp Kota for suggestions on the manuscript improvement. The work performed at Drexel University was supported by the Office of Electricity Delivery and Energy Reliability, Energy Storage Systems Program, through Sandia National Laboratories. The XANES work performed at Brookhaven National Laboratory was supported by the Assistant Secretary for the Energy Efficiency and Renewable Energy Office, Vehicle Technologies, of the U.S. Department of Energy (DOE), under contract No. DE-SC0012704.

Received: ((will be filled in by the editorial staff))

Revised: ((will be filled in by the editorial staff)) Published online: ((will be filled in by the editorial staff))

[1] P. Simon, Y. Gogotsi, Nat. Mater. 2008, 7, 845.

[2] J. Yan, Q. Wang, T. Wei, Z. J. Fan, Adv. Energy Mater. 2014, 4, 43.

[3] S. Hadzi-Jordanov, H. Angerstein-Kozlowska, M. Vukovič, B. E. Conway, J. Electrochem. Soc. 1978, 125, 1471.

[4] a) J. P. Zheng, P. J. Cygan, T. R. Jow, J. Electrochem. Soc. 1995, 142, 2699.

b) S. H. Oh, L. F. Nazar, J. Mater. Chem. 2010, 20, 3834.

[5] C. Sassoye, C. Laberty, H. Le Khanh, S. Cassaignon, C. Boissière, M. Antonietti, C. Sanchez, Adv. Funct. Mater. 2009, 19, 1922.

[6] M. R. Lukatskaya, O. Mashtalir, C. E. Ren, Y. Dall'Agnese, P. Rozier, P. L. Taberna, M. Naguib, P. Simon, M. W. Barsoum, Y. Gogotsi, Science 2013, 341, 1502.

[7] a) M. Naguib, V. N. Mochalin, M. W. Barsoum, Y. Gogotsi, Adv. Mater. 2014, 26, 982; b) K. J. Koski, Y. Cui, ACS Nano 2013, 7, 3739.

[8] M. W. Barsoum, MAX Phases: Properties of Machinable Ternary Carbides and Nitrides, John Wiley \& Sons, 2013.

[9] M. Naguib, M. Kurtoglu, V. Presser, J. Lu, J. Niu, M. Heon, L. Hultman, Y. Gogotsi, M. W. Barsoum, Adv. Mater. 2011, 23, 4248.

[10] J. Halim, M. R. Lukatskaya, K. M. Cook, J. Lu, C. R. Smith, L. A. Naslund, S. J. May, L. Hultman, Y. Gogotsi, P. Eklund, M. W. Barsoum, Chem. Mater. 2014, 26, 2374.

[11] M. Ghidiu, M. R. Lukatskaya, M. Q. Zhao, Y. Gogotsi, M. W. Barsoum, Nature 2014, 516, 78. 
[12] a) M. Naguib, J. Come, B. Dyatkin, V. Presser, P. L. Taberna, P. Simon, M. W. Barsoum, Y. Gogotsi, Electrochem. Commun. 2012, 16, 61; b) M. Naguib, J. Halim, J. Lu, K. M. Cook, L. Hultman, Y. Gogotsi, M. W. Barsoum, J. Am. Chem. Soc. 2013, 135, 15966.

[13] X. Liang, A. Garsuch, L. F. Nazar, Angew. Chem. 2015, 54(13), 3907

[14] Y. Dall'Agnese, M. R. Lukatskaya, K. M. Cook, P. L. Taberna, Y. Gogotsi, P. Simon, Electrochem. Commun. 2014, 48, 118.

[15] M. D. Levi, M. R. Lukatskaya, S. Sigalov, M. Beidaghi, N. Shpigel, L. Daikhin, D. Aurbach, M. W. Barsoum, Y. Gogotsi, Adv. Energy Mater. 2015, 5, 1.

[16] K. Jurewicz, E. Frackowiak, F. Béguin, Appl. Phys. A: Mater. Sci. Process. 2004, 78, 981.

[17] a) J.-K. Chang, M.-T. Lee, W.-T. Tsai, J. Power Sources 2007, 166, 590; b) K.-W. Nam, M. G. Kim, K.-B. Kim, J. Phys. Chem. C 2007, 111, 749.

[18] Y. Mo, M. R. Antonio, D. A. Scherson, J. Phys. Chem. B 2000, 104, 9777.

[19] J. G. Chen, Surf. Sci. Rep. 1997, 30, 1.

[20] Y. Xie, M. Naguib, V. N. Mochalin, M. W. Barsoum, Y. Gogotsi, X. Yu, K. W. Nam, X. Q. Yang, A. I. Kolesnikov, P. R. Kent, J. Am. Chem. Soc. 2014, 136, 6385.

[21] a) W. J. H. Borghols, D. Lutzenkirchen-Hecht, U. Haake, W. Chan, U. Lafont, E. M. Kelder, E. R. H. van Eck, A. P. M. Kentgens, F. M. Mulder, M. Wagemaker, J. Electrochem. Soc. 2010, 157, A582; b) W. J. H. Borghols, D. Lutzenkirchen-Hecht, U. Haake, E. R. H. van Eck, F. M. Mulder, M. Wagemaker, Phys. Chem. Chem. Phys. 2009, 11, 5742. 

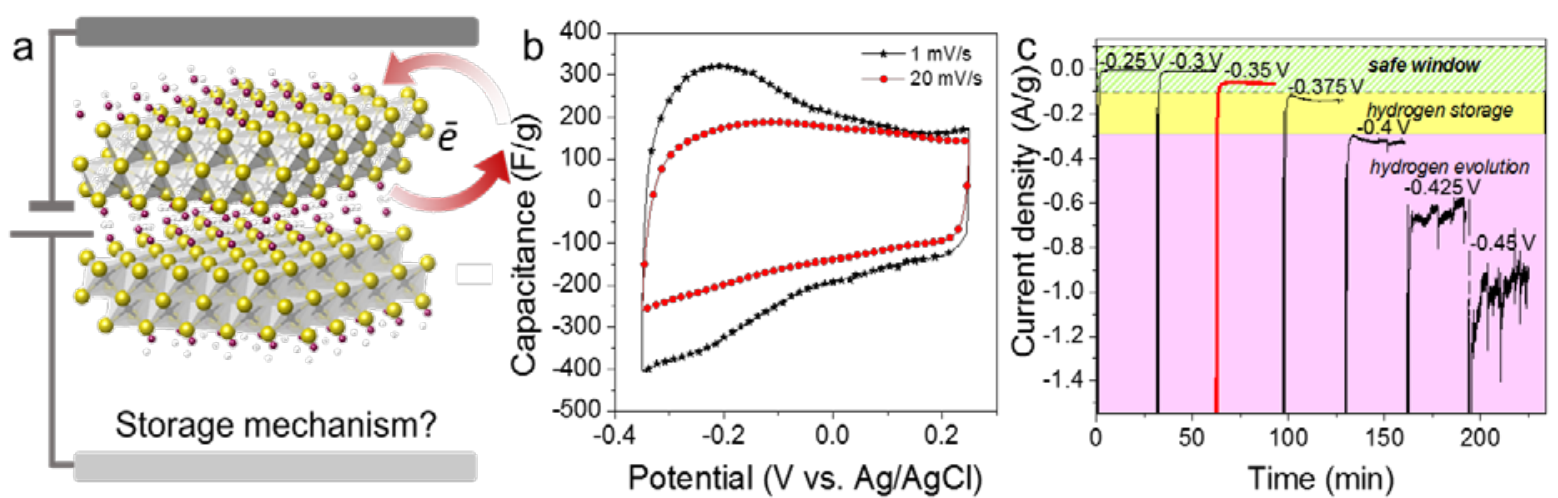

Figure 1. Electrochemical performance of $\mathrm{Ti}_{3} \mathrm{C}_{2} \mathrm{~T}_{\mathrm{x}}$ MXene. a) Schematic of $\mathrm{Ti}_{3} \mathrm{C}_{2}(\mathrm{OH})_{2}$ structure - where atoms of $\mathrm{Ti}$ are yellow, $\mathrm{C}$ are gray and $\mathrm{OH}$ are red - and possible contributions of charge transfer to the capacitance, b) Cyclic voltammetry of $\mathrm{Ti}_{3} \mathrm{C}_{2} \mathrm{~T}_{\mathrm{x}}$ in $1 \mathrm{M}$ $\mathrm{H}_{2} \mathrm{SO}_{4}$ at 1 and $20 \mathrm{mV} / \mathrm{s}$, c) Chronoamperometry data collected at different potentials. 

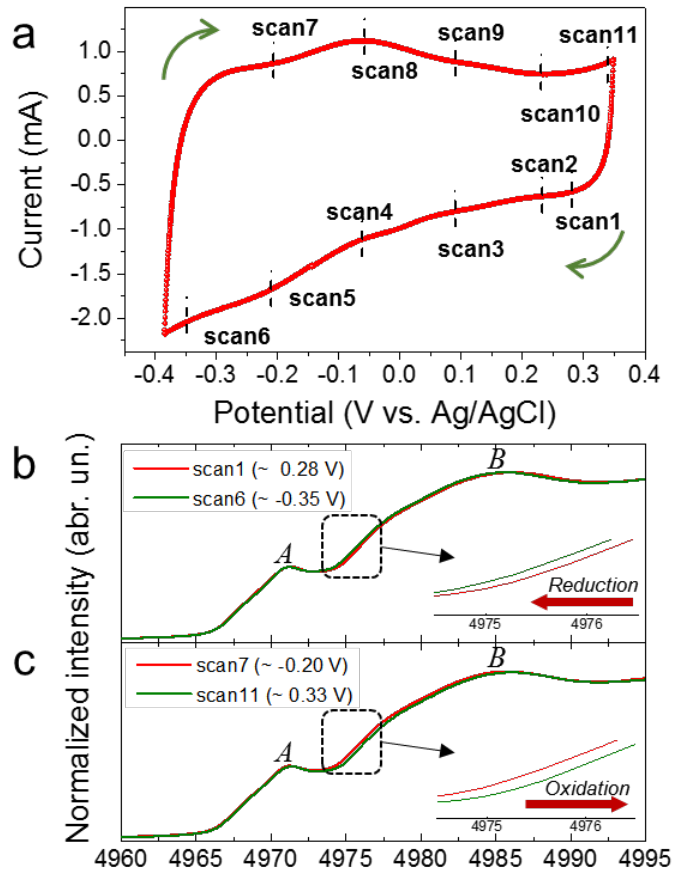

Energy (eV)

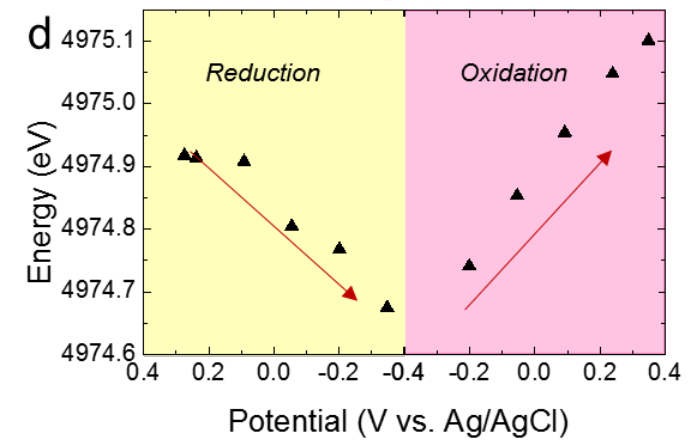

Figure 2. Electrochemical in situ XAS data. a) Cyclic voltammogram collected in in situ XAS electrochemical cell at $1 \mathrm{mV} / \mathrm{s}$ in $1 \mathrm{M} \mathrm{H}_{2} \mathrm{SO}_{4}$ electrolyte. Ti K-edge XANES spectra were collected, b) between 0.3 and $-0.35 \mathrm{~V}$ and, c) between -0.2 to $0.35 \mathrm{~V}$ (vs. Ag/AgCl), d) Variation of Ti edge energy (at half height of normalized XANES spectra) vs. potential during full potential sweep between -0.35 to $0.35 \mathrm{~V}$. 

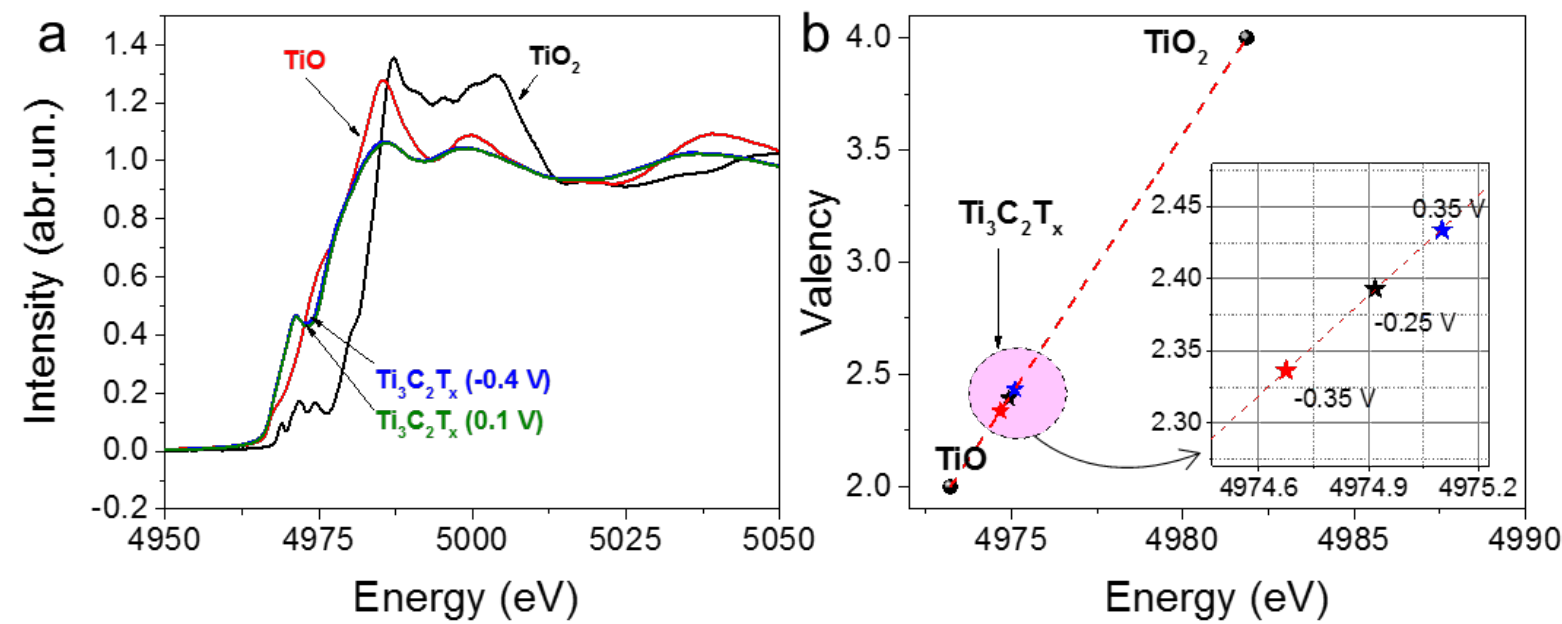

Figure 3. a) Ti K-edge XANES spectra of $\mathrm{Ti}_{3} \mathrm{C}_{2} \mathrm{~T}_{\mathrm{x}}$ at $-0.4 \mathrm{~V}$ (blue line) and $0.1 \mathrm{~V}$ (green line), together with those of $\mathrm{TiO}$ (red line) and $\mathrm{TiO}_{2}$ (black line). b) Average Ti oxidation state determination in $\mathrm{Ti}_{3} \mathrm{C}_{2} \mathrm{~T}_{\mathrm{x}}$ at various applied potentials (see inset), using the Ti K-edge energy shift of the reference $\mathrm{TiO}$ and $\mathrm{TiO}_{2}$ compounds. 


\section{The table of contents entry}

Electrochemical in situ X-ray absorption spectroscopy of 2D titanium carbide MXene was used to probe mechanism of high capacitance of $\mathrm{Ti}_{3} \mathrm{C}_{2} \mathrm{~T}_{\mathrm{x}}$ MXene. Changes in the Ti oxidation state were detected during cycling. This finding confirms that most of the electrochemical behavior of $\mathrm{Ti}_{3} \mathrm{C}_{2} \mathrm{~T}_{\mathrm{x}}$ is due to changes in the oxidation states of the Ti, i.e. pseudocapacitance.

Keywords: capacitance, two-dimensional, MXene, in situ XAS, pseudocapacitance

M. R. Lukatskaya, ${ }^{\dagger}$ S.-M. Bak, ${ }^{\dagger}$ X. Yu, X.-Q. Yang,* M. W. Barsoum, Y. Gogotsi*

Title: Probing the mechanism of high capacitance in two-dimensional titanium carbide using in-situ X-Ray absorption spectroscopy

ToC figure
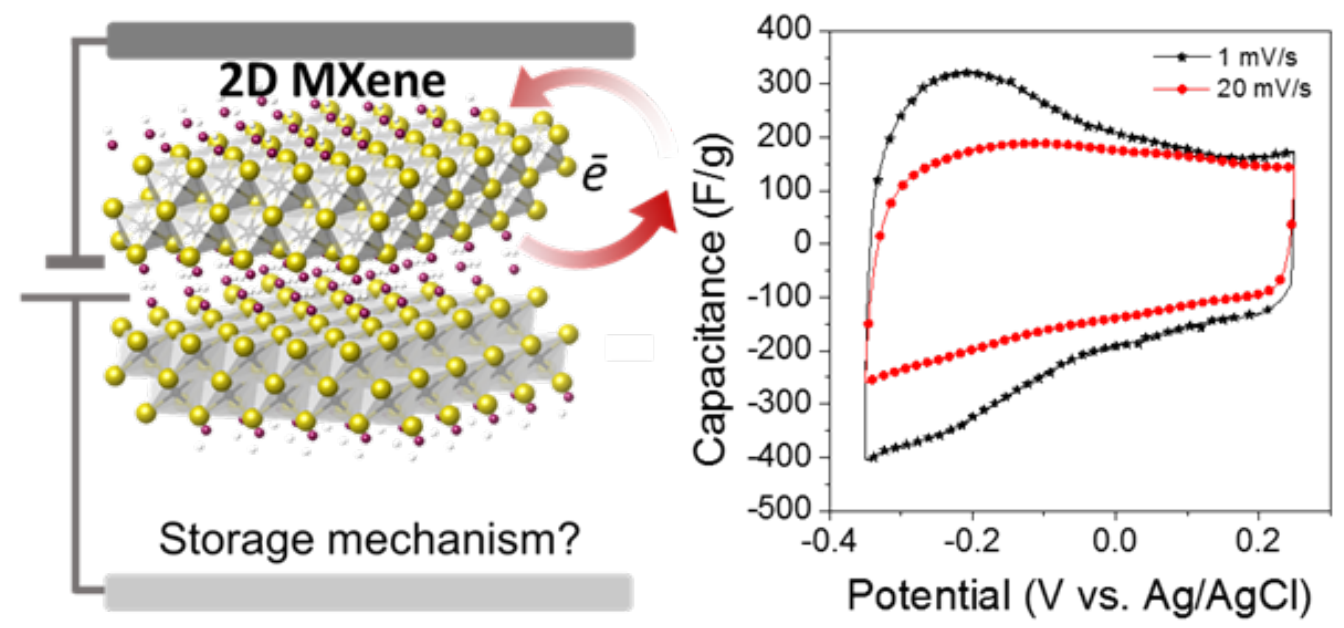
Copyright WILEY-VCH Verlag GmbH \& Co. KGaA, 69469 Weinheim, Germany, 2013.

\section{Supporting Information}

\section{Probing the mechanism of high capacitance in two-dimensional titanium carbide using in-situ X-Ray absorption spectroscopy}

Maria R. Lukatskaya, ${ }^{\dagger}$ Seong-Min Bak, ${ }^{\dagger}$ Xiqian Yu, Xiao-Qing Yang, * Michel W. Barsoum, and Yury Gogotsi*

$\mathrm{Ti}_{3} \mathrm{C}_{2} \mathrm{~T}_{\mathrm{x}}$ MXene synthesis: $30 \mathrm{~mL}$ of $6 \mathrm{M}$ solution of hydrochloric acid (Fisher, technical grade) in distilled water was added to $1.98 \mathrm{~g}$ of $\mathrm{LiF}$ (Alfa Aesar, 98+\%). The mixture was stirred for 5 minutes to dissolve the salt.

Three grams of $\mathrm{Ti}_{3} \mathrm{AlC}_{2}$ (synthesis description can be found elsewhere ${ }^{[\mathrm{S} 1]}$ ) powders were then carefully added, over the course of $10 \mathrm{~min}$., into the LiF containing solution in order to avoid its overheating as a result of the exothermic nature of the reactions. The reaction mixture was then held at $35{ }^{\circ} \mathrm{C}$ for $45 \mathrm{~h}$ under constant stirring, after which the mixture was washed $\sim 5$ times by the addition of distilled water, centrifugation (3500 rpm x 5 minutes for each cycle), and decantation of the supernatant, until the supernatant reached a $\mathrm{pH}$ of approximately 6 . The final powder, with a small amount of water, was filtered through a cellulose nitrate filter $(0.22 \mu \mathrm{m}$ pore size $)$.

$\mathrm{Ti}_{3} \mathrm{C}_{2} T_{x}$ electrode preparation: Electrodes were prepared by mechanical processing of the premixed slurry, containing ethanol (190 proof, Decon Laboratories, Inc.), $\mathrm{Ti}_{3} \mathrm{C}_{2} \mathrm{~T}_{\mathrm{x}}$ powder, polytetrafluoroethylene (PTFE) binder (60 wt.\% in $\mathrm{H}_{2} \mathrm{O}$, Aldrich) and carbon black. The resulting electrodes used for all experiments contained 90 wt. $\%$ of $\mathrm{Ti}_{3} \mathrm{C}_{2} \mathrm{~T}_{\mathrm{x}}, 5$ wt. \% of carbon black, 5 wt. \% of PTFE and were 50-100 $\mu$ m thick with a mass density per unit area of 6-9 $\mathrm{mg} / \mathrm{cm}^{2}$

Activated carbon (AC) counter electrodes: Activated carbon (AC) film electrodes were prepared following the same procedure as for the $\mathrm{Ti}_{3} \mathrm{C}_{2} \mathrm{~T}_{\mathrm{x}}$ electrodes, but without any 
conductive additive. Resulting AC electrodes composition was 95 wt. \% of YP-50 activated carbon (Kuraray, Japan) and 5 wt. \% of the PTFE. They were 100-150 $\mu$ m thick with mass density per unit area of $10-25 \mathrm{mg} / \mathrm{cm}^{2}$.

Cyclic voltammetry was performed using scan rates from $1 \mathrm{mV} / \mathrm{s}$ to $100 \mathrm{mV} / \mathrm{s}$ using a VMP3 potentiostat (Biologic, France). Chronoamperometry data was collected at $-0.35,-0.3,-0.25$, 0.2, -0.15, -0.1, -0.05, 0, 0.05, 0.1, 0.15, 0.2, 0.25, 0.3 V vs. Ag/AgCl.

In-situ X-ray Absorption Spectroscopy (XAS), additional information: Before collecting XAS data, the cell was subjected to ten CV cycles, with scan rate of $10 \mathrm{mV} \mathrm{s}^{-1}$ after which $\mathrm{CV}$ at scan rate of $1 \mathrm{mV} \mathrm{s}^{-1}$ was collected (Fig. 2a). The applied potentials during cyclic voltammetry were held constant for at least 25 min (until a steady-state current was attained) before the in situ XAS measurement and during the XAS signal acquisitions (about $30 \mathrm{~min}$ ) as well. X-ray absorption near edge structure (XANES) spectra were processed using the ATHENA software package. ${ }^{[\mathrm{S} 2]}$ The photoelectron energy origin, $\mathrm{E}_{0}$, was chosen the halfheight of the normalized absorption edge jump.

Reference materials $\mathrm{TiO}$ (99.5\% purity, metals basis) and $\mathrm{TiO} 2$ (99.9\%, metal basis) were purchased from Alfa Aesar.

\section{Supplementary references}

[S1] D. J. Tallman, M. Naguib, B. Anasori, M. W. Barsoum, Scripta Materialia 2012, 66, 805-808.

[S2] B. Ravel, M. Newville, J. Synchrotron Radiation 2005, 12, 537. 